\title{
Iterative Point Matching via Multi-direction Geometric Serialization and Reliable Correspondence Selection
}

\author{
Deheng Qian, Tianshi Chen, Hong Qiao*, Tang Tang \\ D. Qian, H. Qiao and T. Tang are with the State Key Laboratory of Management and Control for Complex Systems, Institute of Automation, Chinese Academy of \\ Sciences, Beijing 100190, China. \\ T. Chen is with the State Key Laboratory of Computer Architecture, Institute of Computing Technology, Chinese Academy of Sciences, Beijing 100190, China.
}

\begin{abstract}
Point matching aims at finding the optimal matching between two sets of feature points. It is widely accomplished by graph matching methods which match nodes of graphs via minimizing energy functions. However, the obtained correspondences between feature points vary in their matching qualities. In this paper, we propose an innovative matching algorithm which iteratively improves the matching found by such methods. The intuition is that we may improve a given matching by identifying "reliable" correspondences, and re-matching the rest feature points without reliable correspondences. A critical issue here is how to identify reliable correspondences, which is addressed with two novel mechanisms, Multi-direction Geometric Serialization (MGS) and Reliable Correspondence Selection (RCS). Specifically, MGS provides representations of the spatial relations among feature points. With these representations, RCS determines whether a correspondence is reliable according to a reliability metric. By recursively applying MGS and RCS, and re-matching feature points without reliable correspondences, a new (intermediate) matching can be obtained. In this manner, our algorithm starts with a matching provided by a classical method, iteratively generates a number of intermediate matchings, and chooses the best one as the final matching. Experiments demonstrate that, our algorithm significantly improves the matching precisions of classical graph matching methods.
\end{abstract}

Keywords: Point matching, order relation, projection, graph matching, dynamic programming.

\section{Introduction}

Point matching plays an important role in computer vision applications such as 3D reconstruction, object classification, video analysis. A popular approach to this problem is to construct a graph model whose nodes represent the feature points and whose edges represent the relations between feature points. Consequently, the matching between two sets of feature points (in a template point set and a deformed scene point set) is accomplished by matching two graphs (namely, a template graph and a deformed scene graph), which provides pairwise correspondences between feature points.

Conventionally, graph matching is formulated as a quadratic assignment problem minimizing a so-called energy function $[18,33]$. However, the quadratic assignment problem is NPcomplete [5, 28], implying that there is no theoretical guarantee that the globally optimal matching can always be found. While this is a bad news for theorists, it sheds some light on the possibility of further improving classical methods in practice. Intuitively, improvements on the matching precision can be achieved by re-matching those feature points whose correspondences are highly likely to be incorrect (i.e., unreliable), but this leads to a critical issue: how to identify reliable or unreliable correspondences.

\footnotetext{
${ }^{*}$ Corresponding author.

Email address: hong.qiao@ia.ac.cn. (Hong Qiao*)
}

In this paper, we propose two novel mechanisms, Multidirection Geometric Serialization (MGS) and Reliable Correspondence Selection (RCS), to address the above issue. For each point set, MGS generates multiple ordering representations of feature points by projecting feature points onto different vectors. For example, after being projected onto a vector, the feature points of a given point set can be serialized and represented as an ordering. By collecting together different ordering representations obtained on different vectors, MGS provides abundant ordering representations for each point set, which describes the relative spatial relations among feature points from different perspectives.

With abundant ordering representations given by MGS, RC$\mathrm{S}$ quantitatively evaluates each correspondence in the original matching, which determines whether the correspondence can be accepted or not. More specifically, let $C^{\mathcal{T}}$ be an ordering representation of the template, $C^{\mathcal{S}}$ be an ordering representation of the scene, and $\left(C^{\mathcal{T}}, C^{\mathcal{S}}\right)$ is called a representation pair. To evaluate whether a given correspondence $[\mathbf{p}, \mathbf{q}]$ is reliable, RCS checks the ordering violation of the correspondence $[\mathbf{p}, \mathbf{q}]$ against other correspondences on each representation pair, and the violation information with respect to multiple representation pairs together determines a reliability metric of the correspondence. Using this metric, the correspondences with the highest reliability are accepted while the rest correspondences are abandoned. The cooperation of MGS and RCS identifies 
those reliable correspondences, and the rest feature points without reliable correspondences will be re-matched by a classical matching method. MGS and RCS, together with the rematching mechanism, generate a new matching that could be better than the initial one.

With the MGS and RCS proposed in this paper, we devise a novel algorithmic framework called Iterative Point Matching (IPM) which starts with the initial matching provided by a classical graph matching method, generates and records a new intermediate matching at each iteration. After a fixed number of iterations, it takes the intermediate matching with the lowest energy as the final matching. The proposed IPM is orthogonal to classical graph matching methods, as it is independent of classical methods, and can be utilized to improve the matching found by many classical methods. In empirical evaluations of the IPM, we use several state-of-the-art matching methods to provide initial matchings for the IPM, and observe that the IP$\mathrm{M}$ significantly improves the matching precision over various datasets.

Our main contributions are three-fold. First, we propose to characterize geometric relations among feature points by projecting the points onto vectors. The obtained ordering representations can precisely characterize the relations given a sufficient number of projection vectors. Second, we propose to iteratively re-match the feature points without reliable correspondences instead of simply abandoning them, which effectively improves the matching quality. Third, the proposed IPM can adapt to many different matching methods to enhance the matching precision, since the IPM is independent of any concrete matching method.

The rest of this paper proceeds as follows: Section 2 presents some related work. Section 3 describes details of the IPM. Section 4 reports the experimental results. Section 5 concludes the whole paper.

\section{Related Work}

Point matching has been studied in various contexts $[8,14$, 17] for its important applications. Besl and McKay proposed a classical algorithm [3] that heuristically corresponds each point in the template to its closest point in the scene. Tsin and Kanade cast the matching problem as finding the maximum kernel correlation configuration of two point sets, where the kernel correlation is defined as a function of the entropy of the point set [29]. Myronenko and Song represented the template points by a Gaussian mixture model such that the matching problem is tackled as a probability density estimation problem [24]. Li et al. reconstructed the location of each feature point by the coordinates of its neighbor points [22], which enables their algorithm to match via linear programming techniques [18]. Zheng and Doermann corresponded points using the similarity of their neighborhood structures, and their algorithm is robust in the presence of non-rigid deformations [32]. Scott and Nowak enforced an order preserving constraint in a contour matching method to regularize the matching process [27]. The above algorithms all exploited the geometric information of feature points.
To leverage more information about the set of feature points, it is a popular approach to build a graph model which is a concise yet informative representation for capturing multiple characteristics of a set of feature points. So, the point matching problem can be solved as a graph matching problem [9]. In general, the graph matching is formulated as a quadratic assignment problem which is known to be NP-complete [5, 28]. Hence, many algorithms aim at tackling this problem more efficiently and precisely [25]. Progressive Graph Matching [7] first matches two small active graphs, and then progressively expands the matching via a probabilistic voting process. Graduated Assignment [15] takes advantage of the Taylor's formula [1] to expand the energy function of the quadratic assignmen$\mathrm{t}$ problem and uses the softassign to gradually obtain the final matching result. Reweighted Random Walk Matching [6] introduces a random walk view on the graph matching problem. The above methods, however, are often trapped by local minima of their energy functions, suggesting that there are still spaces to further improve the matching quality.

So far there have been a number of strategies to improve the matching quality. The statistical robust regression methods, such as LMedS (Least-Median of Squares) [26] and Mestimator [16], try to emulate popular statistical methods, while avoid to be unduly affected by outliers or other small departures from model assumptions. The case diagnostics methods check the influence of putative correspondences on model estimation to reject unreliable correspondences directly. The sampling methods, for example, Random Sample Consensus [13], estimate the parameters of a predefined model by sampling such that correspondences which do not cohere with the estimated model are abandoned. $\mathrm{Li}$ and $\mathrm{Hu}$ proposed to regularize the correspondence function to remove unreliable correspondences [23]. Zhao et al. proposed to use a robust method for vector field learning to identify reliable correspondences [31]. However, previous approaches do not re-match points without reliable correspondences to further improve the matching precision.

In this paper, we propose a new algorithmic framework, the IPM, to improve the matching using the geometric information of points. Our study is substantially different from the above studies, because the IPM not only identifies reliable/unreliable correspondences, but also re-matches points that are identified to be unreliable.

\section{Methodology}

In this section, we elaborate the algorithmic flow of the IPM. We first present the general problem formulation. Then we introduce respectively two novel mechanisms integrated in our IPM framework, namely, Multi-direction Geometric Serialization (MGS) and Reliable Correspondence Selection (RCS), by which reliable correspondences are identified. We also describe the re-matching process where reliable correspondences are exploited to re-match the rest feature points. Finally, We summarize the whole IPM flow. 


\subsection{Problem Formulation}

Suppose the template $\mathcal{T}$ is composed of $n_{t}$ feature points and a deformed scene $\mathcal{S}$ is composed of $n_{s}$ feature points. Let $G_{\mathcal{T}}=$ $\left\{\mathbf{p}_{i}\right\}_{i=1}^{n_{t}}$ and $G_{\mathcal{S}}=\left\{\mathbf{q}_{j}\right\}_{j=1}^{n_{s}}$ be the sets of feature points in $\mathcal{T}$ and $\mathcal{S}$ respectively, where $\mathbf{p}_{i} \in \mathbb{R}^{d}$ is the $i$-th template feature point and $\mathbf{q}_{j} \in \mathbb{R}^{d}$ is the $j$-th scene feature point.

It is a common practice to match some feature points to dummy points to improve the robustness against outliers. Without loss of generality, $n_{s}$ dummy points are induced into $G_{\mathcal{T}}$ and $n_{t}$ dummy points are induced into $G_{\mathcal{S}}$. Hence, $G_{\mathcal{T}}$ and $G_{\mathcal{S}}$ are further extended such that $G_{\mathcal{T}}=\left\{\mathbf{p}_{i}\right\}_{i=1}^{n_{t s}}$, and $G_{\mathcal{S}}=\left\{\mathbf{q}_{j}\right\}_{j=1}^{n_{t s}}$ where $n_{t s}=n_{t}+n_{s}$. Consequently, every feature point in $G_{\mathcal{T}}$ or $G_{\mathcal{S}}$ is matched to one point (real or dummy feature point) in the other set of points.

Let $M(\cdot)$ be a matching function which maps every template feature point $\mathbf{p}_{i}$ to its corresponding feature point. $M(\cdot)$ can be effectively represented by a set of binary variables, more specifically, a matrix $X$ in this paper. Each entry of the matrix is a binary variable:

$$
X_{i j}=\left\{\begin{array}{l}
1, \text { if } M\left(\mathbf{p}_{i}\right)=\mathbf{q}_{j} \\
0, \text { otherwise, }
\end{array}\right.
$$

where $X_{i j}$ is the variable of $X$ at the $i$-th row and $j$-th column. When $X_{i j}=1$, the $i$-th feature point in the template point set is matched with the $j$-th feature point in the scene. $X$ contains exactly one 1 in every row and column.

In classical graph matching methods $[1,6,11,15,20,21$, $30]$, point matching is formulated as a quadratic assignment problem:

$$
\begin{gathered}
\min _{\mathbf{x}} \mathbf{x}^{T} A \mathbf{x} \\
\text { s.t. } \mathbf{1}^{T} X=\mathbf{1}^{T}, X \mathbf{1}=\mathbf{1}, X \in\{0,1\}^{n_{t s} \times n_{t s},}
\end{gathered}
$$

where $\mathbf{x}$ is a binary variable vector concatenating column vectors of matrix $X$, and $\mathbf{1}$ is a column vector with all elements equal to 1 . Entries in matrix $A$ are all non-negative, which measure the dissimilarities between potential correspondences. To be specific, $A_{i j ; a b}$ is the entry of $A$ at the $\left((i-1) n_{t s}+j\right)$-th row and $\left((a-1) n_{t s}+b\right)$-th column that measures the cost of simultaneously matching $\mathbf{p}_{i}$ with $\mathbf{q}_{j}$, and $\mathbf{p}_{a}$ with $\mathbf{q}_{b}$. In other words, $A_{i j ; a b}$ measures the cost of matching edge $\overline{\mathbf{p}_{i} \mathbf{p}_{a}}$ (connecting $\mathbf{p}_{i}$ and $\mathbf{p}_{a}$ in the template $\mathcal{T}$ ) with edge $\overline{\mathbf{q}_{j} \mathbf{q}_{b}}$ (connecting $\mathbf{q}_{j}$ and $\mathbf{q}_{b}$ in the deformed scene $\mathcal{S}$ ). In particular, $A_{i j ; i j}$ measures the unary cost of matching $\mathbf{p}_{i}$ with $\mathbf{q}_{j}$. In case a feature point corresponds to a dummy point, it contributes the cost $\varepsilon$ to the total cost. Detailed descriptions of the matrix $A$ can be found in [33]. By solving the energy function (2), the initial matching $M$ can be obtained.

\subsection{Multi-direction Geometric Serialization}

Based on formulations and notations introduced above, we now present the MGS mechanism which provides abundant ordering representations of sets of feature points.

The ordering representations $\left\{C_{h}^{\mathcal{T}}\right\}_{h=1}^{m}$ of $G_{\mathcal{T}}$ are obtained by projecting feature points in $G_{\mathcal{T}}$ onto different vectors (see Fig. $1)$.
Definition 1. (Serialization Direction) A vector $\boldsymbol{\theta}_{h}^{\mathcal{T}} \in \mathbb{R}^{d}$, onto which a set of feature points are projected, is a serialization direction.

The $h$-th representation $C_{h}^{\mathcal{T}},(h=1,2, \ldots, m)$, results from projecting feature points in $G_{\mathcal{T}}$ onto the $h$-th serialization direction. On each representation $C_{h}^{\mathcal{T}}$, we define a vector $\mathbf{O}_{\mathbf{p}_{i}}^{C_{h}^{\mathcal{T}}}=$

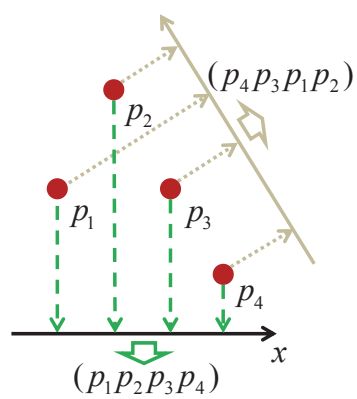

Figure 1: Feature points $p_{1}, p_{2}, p_{3}, p_{4}$ are serialized by projecting them onto multiple serialization directions. On these serialization directions, ordering representations, $\left(p_{1} p_{2} p_{3} p_{4}\right)$ and $\left(p_{4} p_{3} p_{1} p_{2}\right)$, of the point set $\left\{p_{1}, p_{2}, p_{3}, p_{4}\right\}$ can be obtained.

$\left[o_{h}^{\mathcal{T}}\left(\mathbf{p}_{i}, \mathbf{p}_{1}\right), o_{h}^{\mathcal{T}}\left(\mathbf{p}_{i}, \mathbf{p}_{2}\right), \ldots, o_{h}^{\mathcal{T}}\left(\mathbf{p}_{i}, \mathbf{p}_{n_{t s}}\right)\right],\left(i=1,2, \ldots, n_{t s}\right)$, for the point $\mathbf{p}_{i}$ which specifies the order between $\mathbf{p}_{i}$ and any other point in $C_{h}^{\mathcal{T}}$. In case either $\mathbf{p}_{i}$ or $\mathbf{p}_{j}$ is a dummy point, $o_{h}^{\mathcal{T}}\left(\mathbf{p}_{i}, \mathbf{p}_{j}\right)$ is 0 . Otherwise, it is determined by the specific serialization direction:

$$
o_{h}^{\mathcal{T}}\left(\mathbf{p}_{i}, \mathbf{p}_{j}\right)=\left\{\begin{array}{l}
1, \quad \text { if } \boldsymbol{\theta}_{h}^{\mathcal{T}} \cdot\left(\mathbf{p}_{i}-\mathbf{p}_{j}\right) \geq 0 \\
-1, \text { otherwise. }
\end{array}\right.
$$

If $o_{h}^{\mathcal{T}}\left(\mathbf{p}_{i}, \mathbf{p}_{j}\right)=1, \mathbf{p}_{i}$ is greater than or equal to $\mathbf{p}_{j}$ on $C_{h}^{\mathcal{T}}$. Otherwise, $\mathbf{p}_{i}$ is less than $\mathbf{p}_{j}$ on $C_{h}^{\mathcal{T}}$. It is easy to check that all the feature points in $G_{\mathcal{T}}$ can be totally ordered according to the orders determined by (3). We similarly define $\theta_{h^{\prime}}^{\mathcal{S}}, C_{h^{\prime}}^{\mathcal{S}}, \mathbf{O}_{\mathbf{q}_{j}}^{C_{h^{\prime}}^{\mathcal{S}}}$, $\left(h^{\prime}=1,2, \ldots, m^{\prime}\right)$, in $G_{\mathcal{S}}$. The procedure of MGS is illustrated in Algorithm 1.

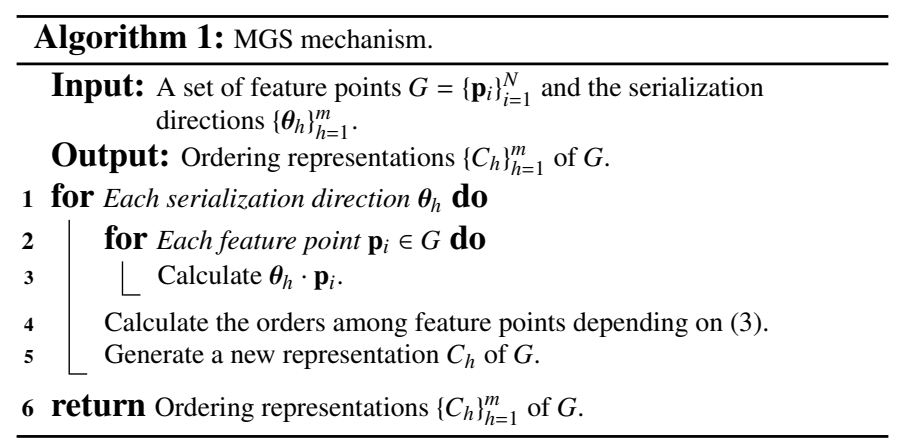

The ordering representations are powerful descriptions of the relative spatial relations among feature points.

Definition 2. (Relative Spatial Relation) Two sets of feature points, $G=\left\{\mathbf{p}_{i}\right\}_{i=1}^{N}$ and $\widetilde{G}=\left\{\mathbf{q}_{j}\right\}_{j=1}^{N}$, have the same relative spatial relations, which is denoted as $G \sim \widetilde{G}$, if there exist constants $s \in \mathbb{R}^{+}, \mathbf{b} \in \mathbb{R}^{d}$ such that for any $\mathbf{p}_{i} \in G, \mathbf{p}_{i}=s \mathbf{q}_{i}+\mathbf{b}$. 
In theory, as the number of serialization directions $m$ increases, the relative spatial relation of $\left\{\mathbf{p}_{i}\right\}_{i=1}^{N}, N \geq 3$, tends to become more precise, as long as $\left\{\mathbf{p}_{i}\right\}_{i=1}^{N}$ are not all collinear.

Yet the non-collinearity is not a necessity in practice. In case all feature points are collinear (which is unlikely given many feature points), the ordering representations are still informative enough to distinguish each feature point from others according to the orders among the feature points.

Besides, MGS has several other favorable characteristics. Firstly, the ordering representations are invariant to scaling and translation. Secondly, the serialization makes it possible to exploit relative spatial relations among feature points that are far from each other. In contrast, classical graph models usually emphasize neighbor structures while deserting a lot of useful global geometric information. Thirdly, the order between two feature points only depends on the feature points themselves as well as the serialization direction. Therefore, they are immune to influences of incorrect correspondences of other feature points.

\subsection{Reliable Correspondence Selection}

Based on MGS, RCS defines the reliability metric of correspondences, and identifies reliable correspondences using the metric. Specifically, after obtaining the initial matching $M$ with classical graph matching methods, we select candidates of reliable correspondences, which is presented in Sections 3.3.1 and 3.3.2. Further, we identify reliable correspondences from the candidates, as detailed in Section 3.3.3.

\subsubsection{Energy Functions}

We select the reliable correspondences according to the orders of feature points on representation pairs.

Definition 3. (Representation Pair) A representation pair $\left(C_{h}^{\mathcal{T}}, C_{h^{\prime}}^{\mathcal{S}}\right)$ is the combination of one ordering representation of $G_{\mathcal{T}}$ and another ordering representation of $G_{\mathcal{S}}$.

The representation pairs used in RCS include all possible combinations of representations of $G_{\mathcal{T}}$ and $G_{\mathcal{S}}$, i.e., $\left\{\left(C_{h}^{\mathcal{T}}, C_{h^{\prime}}^{\mathcal{S}}\right) \mid h=\right.$ $\left.1,2, \ldots, m ; h^{\prime}=1,2, \ldots, m^{\prime}\right\}=\left\{C_{h}^{\mathcal{T}}\right\}_{h=1}^{m} \times\left\{C_{h^{\prime}}^{\mathcal{S}}\right\}_{h^{\prime}=1}^{m^{\prime}}$, which enables our algorithm to be rotation invariant when the serialization directions uniformly distributed over the surface of a $d$ dimensional sphere. On each representation pair $\left(C_{h}^{\mathcal{T}}, C_{h^{\prime}}^{\mathcal{S}}\right)$, we check every pair of feature points $\left(\mathbf{p}_{i}, \mathbf{p}_{j}\right)$ in $C_{h}^{\mathcal{T}}$ to see whether its order $o_{h}^{\mathcal{T}}\left(\mathbf{p}_{i}, \mathbf{p}_{j}\right)$ is the same with $o_{h^{\prime}}^{\mathcal{S}}\left(M\left(\mathbf{p}_{i}\right), M\left(\mathbf{p}_{j}\right)\right)$ in $C_{h^{\prime}}^{\mathcal{S}}$.

Definition 4. (Violation between Correspondences) We say that there is a violation between correspondences, $\left[\mathbf{p}_{i}, M\left(\mathbf{p}_{i}\right)\right]$ and $\left[\mathbf{p}_{j}, M\left(\mathbf{p}_{j}\right)\right]$, on a representation pair $\left(C_{h}^{\mathcal{T}}, C_{h^{\prime}}^{\mathcal{S}}\right)$, if the order of $\left(\mathbf{p}_{i}, \mathbf{p}_{j}\right)$ in $C_{h}^{\mathcal{T}}$ is different from that of $\left(M\left(\mathbf{p}_{i}\right), M\left(\mathbf{p}_{j}\right)\right)$ in $C_{h^{\prime}}^{\mathcal{S}}$, i.e., $o_{h}^{\mathcal{T}}\left(\mathbf{p}_{i}, \mathbf{p}_{j}\right) o_{h^{\prime}}^{\mathcal{S}}\left(M\left(\mathbf{p}_{i}\right), M\left(\mathbf{p}_{j}\right)\right)<0$.

In order to explain the essence of this definition, we present an intuitive example in Fig. 2. A correspondence, e.g., $\left[\mathbf{p}_{4}, \mathbf{q}_{1}\right]$, is intuitively unreliable when it violates too many other correspondences.

We select the candidates of reliable correspondences via minimizing the violations between the selected correspondences on

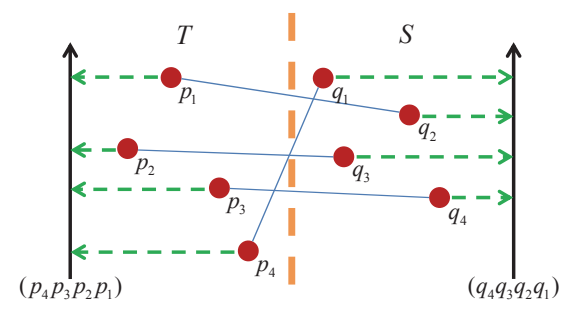

Figure 2: An example of violations between correspondences. The correspondences are indicated by segments linking together two corresponded feature points. $\left[\mathbf{p}_{4}, \mathbf{q}_{1}\right]$ violates all the other correspondences.

each representation pair. To systematically characterize violations on each representation pair, we define multiple energy functions, each of which characterizes the violations on one representation pair. These functions share the same problem formulation of the quadratic assignment problem:

$$
\begin{gathered}
\min _{\widehat{\mathbf{x}}} \widehat{\mathbf{x}}^{T} H^{h h^{\prime} \widehat{\mathbf{x}}} \\
\text { s.t. } \mathbf{1}^{T} \widehat{X}=\mathbf{1}^{T}, \widehat{X} \mathbf{1}=\mathbf{1}, \widehat{X} \in\{0,1\}^{n_{t s} \times n_{t s}},
\end{gathered}
$$

where $\widehat{\mathbf{x}}$ is a binary variable vector concatenating column vectors of matrix $\widehat{X}$. The matrix $\widehat{X}$ indicates the selection of candidates of reliable correspondences. To be specific, let $\widehat{X}_{i j}$ be the variable of $\widehat{X}$ at the $i$-th row and $j$-th column. When $\widehat{X}_{i j}=1$, the correspondence $\left[\mathbf{p}_{i}, \mathbf{q}_{j}\right]$ is selected as a candidate of reliable correspondence. Otherwise, $\left[\mathbf{p}_{i}, \mathbf{q}_{j}\right]$ is not selected. Entries in distance matrix $H^{h h^{\prime}}$ are all non-negative, which measure the violations between correspondences on representation pair $\left(C_{h}^{\mathcal{T}}, C_{h^{\prime}}^{\mathcal{S}}\right)$. To be specific, let $H_{i j ; a b}^{h h^{\prime}}$ be the entry of $H^{h h^{\prime}}$ at the $\left((i-1) n_{t s}+j\right)$-th row and $\left((a-1) n_{t s}+b\right)$-th column which represents whether there is a violation between $\left[\mathbf{p}_{i}, \mathbf{q}_{j}\right]$ and $\left[\mathbf{p}_{a}, \mathbf{q}_{b}\right]$,

$$
H_{i j ; a b}^{h h^{\prime}}=\left\{\begin{array}{c}
0, \text { if }\left(\begin{array}{c}
o_{h}^{\mathcal{T}}\left(\mathbf{p}_{i}, \mathbf{p}_{a}\right) o_{h^{\prime}}^{\mathcal{S}}\left(\mathbf{q}_{j}, \mathbf{q}_{b}\right) \geq 0 \\
M\left(\mathbf{p}_{i}\right)=\mathbf{q}_{j} \\
M\left(\mathbf{p}_{a}\right)=\mathbf{q}_{b}
\end{array}\right) \\
1, \text { otherwise. }
\end{array}\right.
$$

The matrix $H^{h h^{\prime}},\left(h=1,2, \ldots, m ; h^{\prime}=1,2, \ldots, m^{\prime}\right)$, connects the initial matching with RCS. $H^{h h^{\prime}}$ can be extracted from the representation pair $\left(C_{h}^{\mathcal{T}}, C_{h^{\prime}}^{\mathcal{S}}\right)$ provided by MGS, and offers information to select candidates of reliable correspondences.

Note that (5) implies that we set the cost of matching any feature point with dummy point to 0 . In order to accept correspondences between real feature points as many as possible, we further modify the energy function (4) as:

$$
\begin{gathered}
\min _{\widehat{\mathbf{x}}} \widehat{\mathbf{x}}^{T} H^{h h^{\prime}} \widehat{\mathbf{x}}-w \sum_{i=1}^{n_{t}} \sum_{j=1}^{n_{s}} \widehat{X}_{i j} \\
\text { s.t. } \mathbf{1}^{T} \widehat{X}=\mathbf{1}^{T}, \widehat{X} \mathbf{1}=\mathbf{1}, \widehat{X} \in\{0,1\}^{n_{t s} \times n_{t s}},
\end{gathered}
$$

where $w>0$ is a weight. The term $\sum_{i=1}^{n_{t}} \sum_{j=1}^{n_{s}} \widehat{X}_{i j}$ refers to the number of selected candidates of reliable correspondences between real feature points. 


\subsubsection{Longest Common Subsequences}

The energy function (6) shares the problem formulation of the quadratic assignment problem, which is known to be NPcomplete [5, 28]. Brute searching methods such as branch and bound [4] are computationally expensive, thus are not applicable to large-scale problems. As mentioned in Section 2, many methods try to find efficient algorithms to approximately solve this problem. However, they are often trapped by local minima. While the quadratic assignment problem is generally NPcomplete, we discover in this paper that a special case of the energy function (6) is actually polynomial-time solvable. We also present the detailed algorithm of finding the global optimum of the special case of the energy function (6) as illustrated below.

As a special case of the energy function, the weight $w$ is set to be small enough

$$
0<w<\frac{1}{n_{t} n_{s}},
$$

so that the cost of any violation among selected candidates of reliable correspondences is higher than the benefit obtained by selecting more correspondences between real feature points. The energy function (6) is further modified as:

$$
\begin{aligned}
& \min _{\widehat{\mathbf{x}}} \widehat{\mathbf{x}}^{T} H^{h h^{\prime}} \widehat{\mathbf{x}}-w \sum_{i=1}^{n_{t}} \sum_{j=1}^{n_{s}} \widehat{X}_{i j} \text {, where } 0<w<\frac{1}{n_{t} n_{s}}, \\
& \text { s.t. } \mathbf{1}^{T} \widehat{X}=\mathbf{1}^{T}, \widehat{X} \mathbf{1}=\mathbf{1}, \widehat{X} \in\{0,1\}^{n_{t s} \times n_{t s}} \text {. }
\end{aligned}
$$

Hence, the minimization process of energy function (8) is dominated by the term $\widehat{\mathbf{x}}^{T} H^{h h^{\prime}} \widehat{\mathbf{x}}$. However, considering $\widehat{\mathbf{x}}^{T} H^{h h^{\prime}} \widehat{\mathbf{x}}$ alone, its global optima cannot accept any violation. In other words, the global optima of $\widehat{\mathbf{x}}^{T} H^{h h^{\prime}} \widehat{\mathbf{x}}$ can be any combination of correspondences which do not violate each other on the representation pair $\left(C_{h}^{\mathcal{T}}, C_{h^{\prime}}^{\mathcal{S}}\right)$. In a degenerate case, the global optima of $\widehat{\mathbf{x}}^{T} H^{h h^{\prime}} \widehat{\mathbf{x}}$ can be achieved by matching all feature points to dummy points. The second term $-w \sum_{i=1}^{n_{t}} \sum_{j=1}^{n_{s}} \widehat{X}_{i j}$ addresses this issue. Concretely, it chooses the final solution from global optima of $\widehat{\mathbf{x}}^{T} H^{h h^{\prime}} \widehat{\mathbf{x}}$ to select as many as possible the correspondences between real feature points. As a result, optimizing the energy function is converted to a Longest Common Subsequence (LCS) problem, which is illustrated below.

The LCS problem selects the largest set of common elements of two sequences while requiring the selected elements to have the same order in the two sequences (e.g., the LCS of letter sequences $(a b c d)$ and $(d a e c)$ is $(a c))$. Given one sequence with $n_{t}$ elements and another sequence with $n_{s}$ elements, the LCS of such two sequences is optimally computed by dynamic programming [10] with the time complexity $O\left(n_{t} n_{s}\right)$.

Recall that, in MGS, the ordering representations, namely, $\left\{C_{h}^{\mathcal{T}}\right\}_{h=1}^{m}$ and $\left\{C_{h^{\prime}}^{\mathcal{S}}\right\}_{h^{\prime}=1}^{m^{\prime}}$, can be regarded as sequences and each corresponded feature point in $G_{\mathcal{T}}$ has a partner in $G_{\mathcal{S}}$. Here, we define the "longest common subsequence" of ordering representations $C_{h}^{\mathcal{T}}$ and $C_{h^{\prime}}^{\mathcal{S}}$.

Definition 5. (Longest Common Subsequence of A Representation Pair) The longest common subsequence of a representation pair $\left(C_{h}^{\mathcal{T}}, C_{h^{\prime}}^{\mathcal{S}}\right)$ is the longest sequence of correspondences, $\left(\left[\mathbf{p}_{i}, M\left(\mathbf{p}_{i}\right)\right],\left[\mathbf{p}_{j}, M\left(\mathbf{p}_{j}\right)\right], \ldots,\left[\mathbf{p}_{l}, M\left(\mathbf{p}_{l}\right)\right]\right)$, in which there is no violation between any pair of correspondences.

For example, if correspondences $\left[\mathbf{p}_{i}, M\left(\mathbf{p}_{i}\right)\right]$ and $\left[\mathbf{p}_{j}, M\left(\mathbf{p}_{j}\right)\right]$ are in the LCS of the representation pair $\left(C_{h}^{\mathcal{T}}, C_{h^{\prime}}^{\mathcal{S}}\right)$, the order of $\left(\mathbf{p}_{i}, \mathbf{p}_{j}\right)$ in $C_{h}^{\mathcal{T}}$ is the same with that of $\left(M\left(\mathbf{p}_{i}\right), M\left(\mathbf{p}_{j}\right)\right)$ in $C_{h^{\prime}}^{\mathcal{S}}$, i.e., $o_{h}^{\mathcal{T}}\left(\mathbf{p}_{i}, \mathbf{p}_{j}\right) o_{h^{\prime}}^{\mathcal{S}}\left(M\left(\mathbf{p}_{i}\right), M\left(\mathbf{p}_{j}\right)\right) \geq 0$.

Based on Definition 5, the LCS of a representation pair provides exactly what we want by minimizing the energy function (8). In theory, the correspondences in the LCS of $\left(C_{h}^{\mathcal{T}}, C_{h^{\prime}}^{\mathcal{S}}\right)$ together construct the global optimum of the energy function (8) defined on $\left(C_{h}^{\mathcal{T}}, C_{h^{\prime}}^{\mathcal{S}}\right)$, as stated in Property 1 (see the proof in Appendix A).

Property 1. Given one representation pair, $\left(C_{h}^{\mathcal{T}}, C_{h^{\prime}}^{\mathcal{S}}\right)$, the energy function (8) defined on $\left(C_{h}^{\mathcal{T}}, C_{h^{\prime}}^{\mathcal{S}}\right)$ has the optimum $X^{*}$ which indicates the correspondences in the LCS of $\left(C_{h}^{\mathcal{T}}, C_{h^{\prime}}^{\mathcal{S}}\right)$.

Property 1 provides a geometric insight on the energy function (8). Note that a relatively long LCS imposes more ordering constraints on its correspondences than a short LCS does. While incorrect correspondences generally satisfy only few ordering constraints because of their own disorders (see Fig. 2 for instance). Hence incorrect correspondences may especially appear in some relatively short common subsequences. In contrast, they would only accidentally appear in a long common subsequence. And the longer the common subsequence is, the more likely the correspondences comprising it are correct (see Section 4.1). Consequently, it is reasonable to find the LCSes to provide candidates of reliable correspondences.

Property 1 reveals that the global optimum of the energy function (8) can be efficiently obtained by some polynomialtime algorithms [10]. In contrast, the general quadratic assignment problem can only be efficiently solved with an approximate optimal solution by existing algorithms.

Based on Property 1, multiple energy functions (8) defined on different representation pairs can be efficiently solved, providing candidates of reliable correspondences. Then, reliable correspondences can be identified from these candidates.

\subsubsection{Voting by Top-k LCSes}

Among the candidates of reliable correspondences in the LCSes, RCS, which is summarized in Algorithm 2, identifies correspondences that are most reliable according to a reliability metric. The reliability metric of a given correspondence is defined as the number of ballots received in a voting process detailed below.

The voting process evaluates comprehensively each correspondence with two steps. At the first step, we find the top- $k$ longest LCSes, endow them voting ballots, and abandon all the rest LCSes. As analysed previously, we prefer relatively long LCSes because the longer an LCS is, the more likely the correspondences comprising it are correct.

At the second step, we let the top- $k$ longest LCSes vote for their included correspondences. In other words, a correspondence receives one ballot from each of the LCSes including this correspondence. The number of ballots received by the correspondence is regarded as the reliability measurement of the 


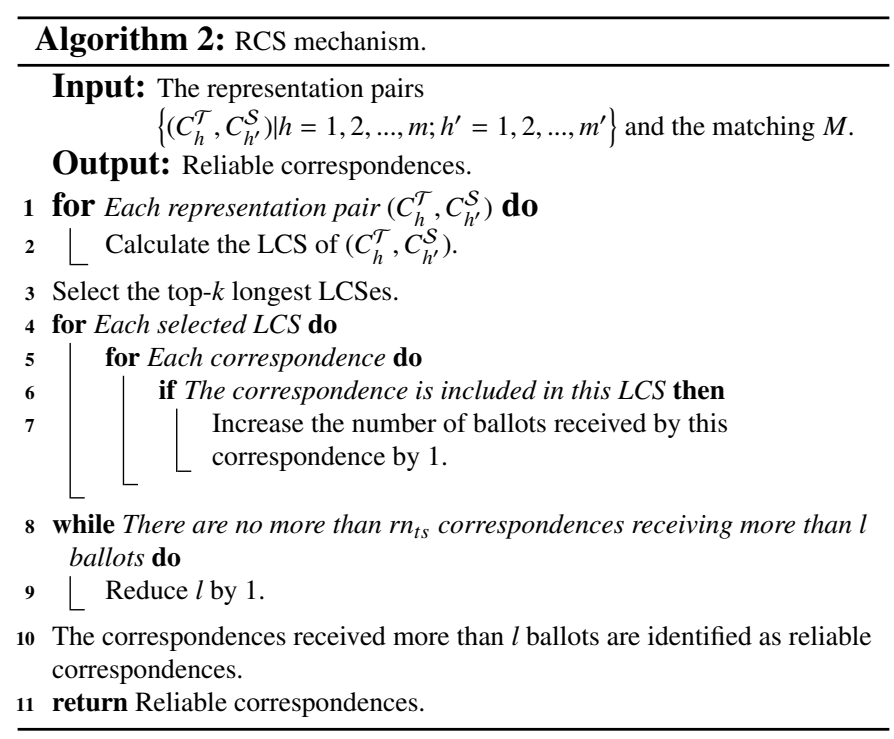

correspondence. Finally, correspondences received more than $l$ ballots are identified as reliable correspondences. Sometimes there are not sufficient reliable correspondences, thus we iteratively reduce the threshold $l$ by one, until there have been more than $r n_{t s}$ correspondences receiving more than $l$ ballots, where $n_{t s}$ is the total number of correspondences and $r \in[0,1]$ is a ratio. In the degenerate case $(r=1)$, RCS identifies all correspondences as reliable ones.

The identified reliable correspondences are then accepted as a part of an intermediate matching. Accordingly, the feature points with reliable correspondences are removed from the feature point sets $G_{\mathcal{T}}$ and $G_{\mathcal{S}}$ respectively. The remaining feature points are without reliable correspondences, thus need to be rematched for a potentially better matching.

\subsection{Re-matching Process}

The re-matching process is tackled as a new matching problem at a smaller scale than the original one. For the ease of notation, let $\left\{\widehat{\mathbf{p}}_{i}\right\}_{i=1}^{n}$ be feature points with reliable correspondences in the template $\mathcal{T}$. In the same point set, let $\left\{\mathbf{p}_{i}\right\}_{i=1}^{n_{t s}-n}$ be the set of remaining points without reliable correspondences, where $n_{t s}$ is the total number of points. Similarly, we define $\left\{\widehat{\mathbf{q}}_{i}\right\}_{i=1}^{n}$ and $\left\{\mathbf{q}_{i}\right\}_{i=1}^{n_{t s}-n}$ for the scene $\mathcal{S}$.

To match $\left\{\mathbf{p}_{i}\right\}_{i=1}^{n_{t s}-n}$ and $\left\{\mathbf{q}_{i}\right\}_{i=1}^{n_{t s}-n}$ in the re-matching process, we must calculate a new distance matrix $A^{*}$ which is used in an energy function similar to (2) while substituting $A^{*}$ for the distance matrix $A$ in (2). To be specific, $A^{*}$ can be calculated as the sum of two parts which are described below.

The first part of $A^{*}$, which is denoted by $A^{\prime}$, reflects the dissimilarities between $\left\{\mathbf{p}_{i}\right\}_{i=1}^{n_{t s}-n}$ and $\left\{\mathbf{q}_{i}\right\}_{i=1}^{n_{t s}-n}$. We can easily obtain $A^{\prime}$ with $\left(n_{t s}-n\right)^{2}$ rows and $\left(n_{t s}-n\right)^{2}$ columns by deleting rows and columns of the distance matrix $A$ with $n_{t s}^{2} \times n_{t s}^{2}$, which is used in the initial matching. The deleted rows and columns are related to $\left\{\widehat{\mathbf{p}}_{i}\right\}_{i=1}^{n}$ and $\left\{\widehat{\mathbf{q}}_{i}\right\}_{i=1}^{n}$.

The second part of $A^{*}$, which is denoted by $\Lambda$, derives from the geometric relations between the remaining feature points and those with reliable correspondences. Specifically, we extract a new kind of local features from feature points without reliable correspondences, using their geometric positions relative to feature points with reliable correspondences. This kind of local features is called the correspondence context in the rest of the paper.

Correspondence context exploits the property that the position of a feature point can be located by its Euclidian distances to no less than three non-collinear feature points having reliable correspondences. Let $\mathbf{p}_{i}=\left(x_{i}, y_{i}\right)^{T}$ be the $i$-th remaining feature point in the template $\mathcal{T}$. Then, the position of $\mathbf{p}_{i}$ is located by its distances to $\widehat{\mathbf{p}}_{1}, \widehat{\mathbf{p}}_{2}, \ldots, \widehat{\mathbf{p}}_{n}$. Let $e_{\mathbf{p}_{i} \widehat{\mathbf{p}}_{v}}=\left\|\mathbf{p}_{i}-\widehat{\mathbf{p}}_{v}\right\|,(v=1,2, \ldots, n)$, be the Euclidian distance between $\mathbf{p}_{i}$ and $\widehat{\mathbf{p}}_{v}$. We can form a distance vector $\mathbf{D}_{\mathbf{p}_{i}}=\left(e_{\mathbf{p}_{i} \widehat{\mathbf{p}}_{1}}, e_{\mathbf{p}_{i} \widehat{\mathbf{p}}_{2}}, \ldots, e_{\mathbf{p}_{i} \widehat{\mathbf{p}}_{n}}\right)$ which is represented as histograms. The histograms $\mathbf{D}_{\mathbf{p}_{i}}$ are assigned to $\mathbf{p}_{i}$ as its correspondence context.

Assuming the deformation between $\mathcal{T}$ and $\mathcal{S}$ is mainly rigid, the correspondence context of $\mathbf{p}_{i}$ in $\mathcal{T}$ should be similar to that of the counterpart of $\mathbf{p}_{i}$ in $\mathcal{S}$. The dissimilarity measurement of the correspondence context is defined below using the $\chi^{2}$ test statistic:

$$
\zeta\left(\mathbf{p}_{i}, \mathbf{q}_{j}\right)=\sum_{v=1}^{n} \frac{\left(e_{\mathbf{p}_{i} \widehat{\mathbf{p}}_{v}}-e_{\mathbf{q}_{j} M\left(\widehat{\mathbf{p}}_{v}\right)}\right)^{2}}{e_{\mathbf{p}_{i} \widehat{\mathbf{p}}_{v}}+e_{\mathbf{q}_{j} M\left(\widehat{\mathbf{p}}_{v}\right)}},
$$

where $\mathbf{p}_{i} \in G_{\mathcal{T}}, \mathbf{q}_{j} \in G_{\mathcal{S}}$ are feature points without reliable correspondence and $\zeta\left(\mathbf{p}_{i}, \mathbf{q}_{j}\right)$ is the dissimilarity between $\mathbf{p}_{i}$ and $\mathbf{q}_{j}$ in their correspondence contexts. The pairwise dissimilarity values form diagonal elements of the distance matrix $\Lambda$ having $\left(n_{t s}-n\right)^{2}$ rows and $\left(n_{t s}-n\right)^{2}$ columns. To be specific, $\Lambda_{i j ; i j}$ (the entry of $\Lambda$ at the $\left((i-1)\left(n_{t s}-n\right)+j\right)$-th row and $\left((i-1)\left(n_{t s}-n\right)+j\right)$ th column $)$ is calculated as $\zeta\left(\mathbf{p}_{i}, \mathbf{q}_{j}\right)$. The rest elements of $\Lambda$ are zeros.

With $A^{\prime}$ and $\Lambda$, we calculate the new matrix $A^{*}$ as

$$
A^{*}=A^{\prime}+\lambda \Lambda,
$$

where $\lambda \geq 0$ is a weight controlling the strength of the influence of reliable correspondences on the re-matching process. A large $\lambda$ is preferable when the deformation between $\mathcal{T}$ and $\mathcal{S}$ is mainly rigid, since the correspondence context, which exploits pairwise Euclidian distances between feature points, works the best under such deformation.

\subsection{IPM Framework}

In this section, we summarize the framework of the proposed IPM, whose algorithmic flow is illustrated in Algorithm 3.

At the very beginning, we establish the initial matching with a graph matching method. Then, we apply the MGS mechanis$\mathrm{m}$ to gain ordering representations of the sets of feature points. Based on these representations, we conduct the RCS mechanism to select reliable correspondences. The feature points with reliable correspondences are not matched again in the rematching process, which focuses only on the feature points without reliable correspondences. The process "MGS + RCS + Re-matching" is called an inner loop (from step 5 to step 8 in Algorithm 3) in IPM, which produces a part of an intermediate matching. After a sufficient number of inner loops, all 


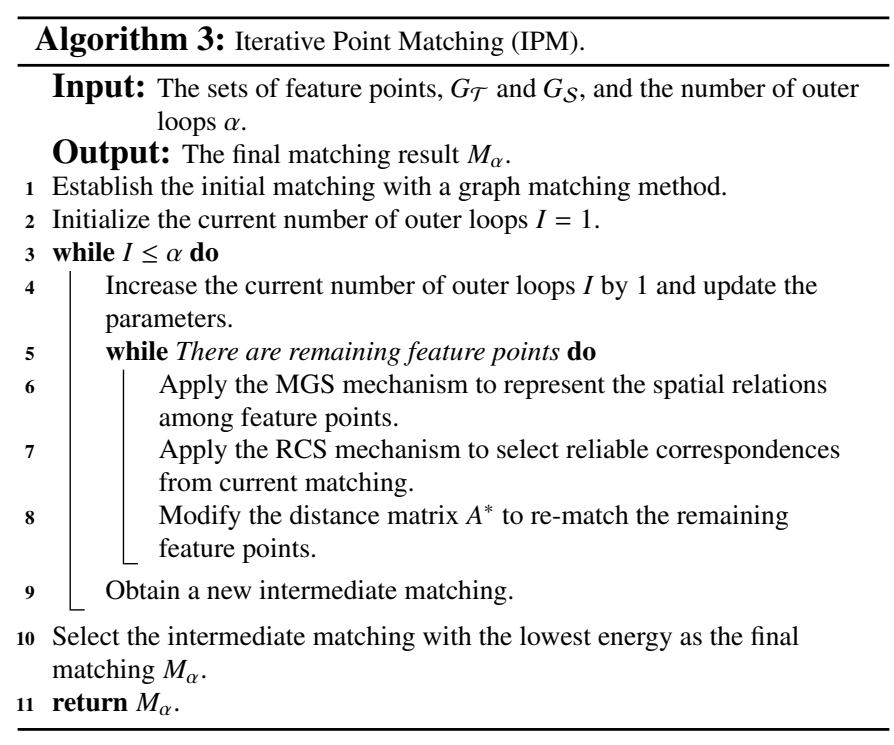

feature points can be reliably corresponded, resulting in an intermediate complete matching, where the complete matching is intermediate in the sense that it is not the final matching output by the IPM. The process of obtaining an intermediate complete matching is called an outer loop (from step 3 to step 9 in Algorithm 3) in IPM.

The number of total outer loops in IPM is denoted by $\alpha$. In each outer loop, a correspondence is not changeable once being identified as a reliable one. Hence, the obtained intermediate matching heavily depends on the initial matching. If the initial matching has a very low precision, the matching precision of the intermediate matching derived in one outer loop could not be good. To alleviate this problem, we use the current intermediate matching as the input of another outer loop to obtain a new intermediate matching. In an iterative manner, the matching precision is further improved.

As summarized in Algorithm 3, each outer loop generates an intermediate complete matching. The final matching is therefore selected from all the $\alpha$ intermediate matchings according to their energies. Their energies are calculated according to the energy function (2) with the distance matrix $A$ which is used in the initial matching process. The matching with the lowest energy is chosen as the final matching.

\section{Experiments}

In this section, we evaluate the IPM on the following datasetS.

- Chinese character and fish dataset [8]. This dataset includes two subsets, i.e., a Chinese character subset and a fish subset. Each subset includes 500 pairs of point set$\mathrm{s}$ with non-rigid deformations and 600 pairs of point sets with noise pollution. Every 100 pairs of point sets have the same level of deformation or noise pollution. Each set includes 98 to 105 points, and the matching ground truth of each pair of sets is available.
- PASCAL image dataset [21]. This dataset consists of real images selected from PASCAL 2007 [12], including 30 pairs of car images and 20 pairs of motorbike images. Each pair of images contains 15 to 52 manually labeled correspondences and several outliers.

- Horse silhouette dataset. This dataset consists of 299 frames of a horse silhouette. Each frame contains 35 manually labeled feature points with matching ground truth.

- 3D Face 50 dataset. This dataset includes 50 3D point sets, each of which is sampled from a human face. Each point set includes 392 points with matching ground truth.

- Synthetic 3D dataset. This dataset includes 2000 3D matching problems. Every 500 problems have the same level of noise pollution. Each point set in a matching problem includes 100 points.

- CMU house and hotel dataset. This dataset includes 111 frames of a house and 101 frames of a hotel. Each frame contains 30 manually labeled feature points with matching ground truth.

Major challenges of these datasets include non-rigid deformations, noise pollution, outliers, and different kinds of affine transformations.

To increase the reproductivity, we elaborate parameter settings used in our experiments. The current setting of parameters is obtained after intensive experiments on multiple datasets. Due to the limited space, here we do not elaborate the details, but directly specify the concrete setting instead. To be specific, the numbers of serialization directions for template and scene point sets, namely, $m$ and $m^{\prime}$, are set to be the same, i.e., $m=m^{\prime}$. In both $2 \mathrm{D}$ and 3D matching problems, we set $m=32$ (see Section 4.2.5). In 2D cases, the serialization directions $\left\{\boldsymbol{\theta}_{h}^{\mathcal{T}}\right\}_{h=1}^{m}$ and $\left\{\boldsymbol{\theta}_{h^{\prime}}^{\mathcal{S}}\right\}_{h^{\prime}=1}^{m^{\prime}}$ uniformly spread in the interval $[0,2 \pi)$. In 3D cases, serialization directions are from the centroid of a regular dodecahedron to 20 vertices of the dodecahedron, or 12 middle points in surfaces of the dodecahedron. The number of votable LCSes, $k$, is set to 40 . The threshold $l$, which specifies the least number of ballots that a reliable correspondence must receive, is set to 32. The parameter $r$ controlling the proportion of reliable correspondences in each inner loop is set to 0.1 in Section 4.2.4 and Section 4.2.5 to gain better efficiency on matching problems with a relatively large size in the dataset, but is kept to be 0 in all the rest experiments. The weight $\lambda$ in Eq. (10), which controls the strength of reliable correspondences' influence on re-matching processes, is set to 0.1 .

Under the above settings, we evaluate and compare different algorithms in terms of their matching error rates, which is defined as the ratio between the number of incorrect correspondences of inliers and the total number of inliers.

\subsection{A Close Look at LCSes and Voting in IPM}

LCSes play a central role in the IPM flow, not only because longer LCSes are likely to contain more reliable correspondences, but also because they collaboratively specify which 
points must be re-matched via a voting process. Here we empirically validate the effectiveness of the building blocks, i.e., LCSes and the voting processes, to support the promising performance of IPM showed in later subsections.

We run IPM on four typical graph matching problems randomly picked from the Chinese character-deformation subdataset, the fish-deformation sub-dataset, the horse silhouette dataset and the CMU hotel sub-dataset. The first three of them incorporate non-rigid deformations (see Fig. 3-(a)).

Without losing any generality, the IPM evaluated here uses a classic matching method, Graduated Assignment (GA) [15] (see Section 4.2 for more details). We report correlations between the lengths and qualities of LCSes in Fig. 3-(b), where the quality of an LCS is measured by the ratio of correct correspondences induced by the $\operatorname{LCS}^{1}$. For both rigid and non-rigid cases, it can be observed that a longer LCS in general has a better quality, and top- $k(k=40)$ LCSes tend to become longer as the IPM proceeds (see Fig. 3-(c)).

We also quantitatively evaluate the voting processes in IPM. To be specific, we record the number of ballots received by correct and incorrect correspondences in the first inner loop of the first five outer loops, and illustrate the data statistics in Fig. 3(d). We observe that correct correspondences generally receive more ballots than incorrect ones do. Hence, correct correspondences generally have higher chances to survive in the voting processes. It is this preference on correct correspondences that drives IPM to gradually find better matchings.

\subsection{IPM Performance}

In this subsection, we conduct comprehensive experiments to evaluate the IPM on multiple datasets. We evaluate two different implementations of the IPM, in which we respectively embed two state-of-the-art graph matching methods into the general IPM flow (see Algorithm 3) to conduct the initial matching and re-matching processes. These methods are Graduated Assignment (GA) [15] and Reweighted Random Walk Matching (RRWM) [6]. In the context of the IPM, we call them base matching methods which can be integrated in the IPM framework. For 2D matching problems, graph models to be matched are built with Delaunay triangulation [19]. Features of a node are set to be its shape contexts [2], and features of an edge are set according to its length and angles between this and other edges, following the settings used in [21]. In solving 3D matching problems, however, we build graph models by linking every node to its nearest $K$ nodes, where $K=5$. Besides, we do not use any node feature but only use edge features.

\subsubsection{Chinese Character and Fish Dataset}

We first evaluate the IPM on Chinese character and fish dataset [8], where the deformation level and noise level are quantified following [8, 32]. We compare the initial matching with the final matchings founded after $\alpha$ outer loops of

${ }^{1}$ For example, we consider an LCS induces two correspondences $\left(\left[\mathbf{p}_{1}, \mathbf{q}_{1}\right],\left[\mathbf{p}_{2}, \mathbf{q}_{2}\right]\right)$. If $\left[\mathbf{p}_{1}, \mathbf{q}_{1}\right]$ is the only correct correspondence here, the ratio of correct correspondences induced by this LCS is $1 / 2=0.5$.
IPM over the whole dataset, and we try three settings of $\alpha$ (1, 3, and 10). We report the results in Figs. 4 (Chinese character-deformation sub-dataset), 5 (Chinese character-noise sub-dataset), 6 (fish-deformation sub-dataset) and 7 (fish-noise sub-dataset). In these Figs., we let, for example, IPM(RRWM, 3) denote the IPM using 3 outer loops to improve the initial matching found by RRWM.

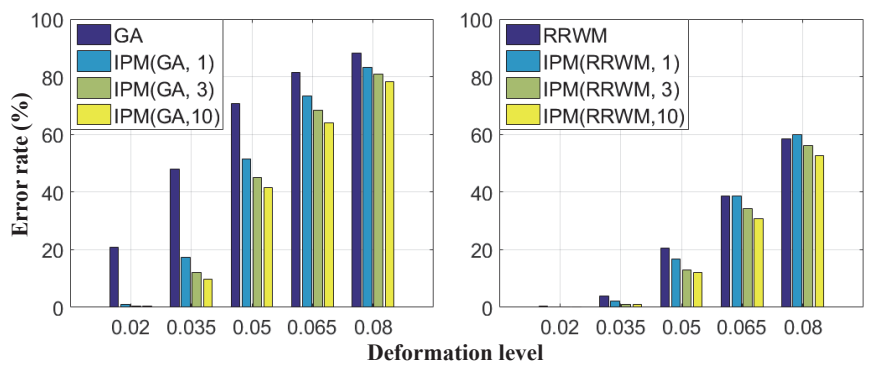

Figure 4: Error rates on the Chinese character-deformation sub-dataset.
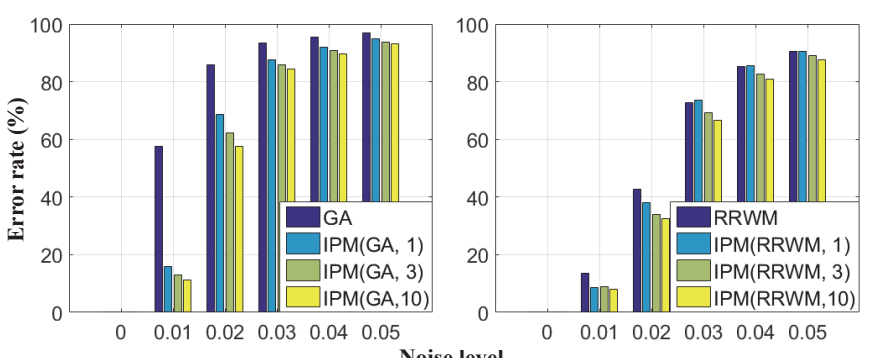

Figure 5: Error rates on the Chinese character-noise sub-dataset.

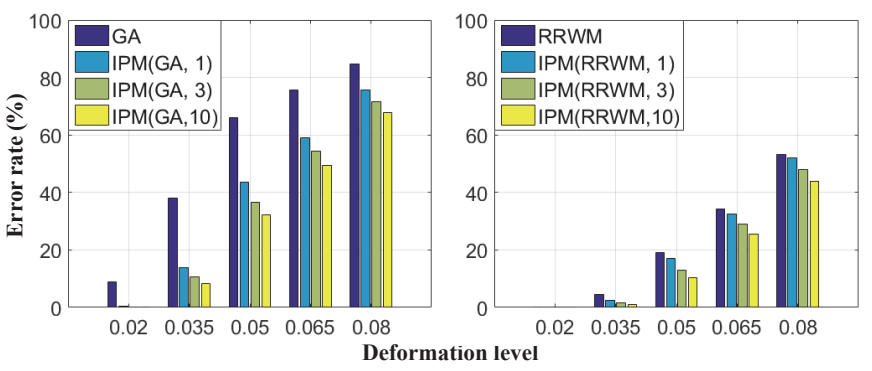

Figure 6: Error rates on the fish-deformation sub-dataset.

We observe from these figures that the final matching quality relies on the base matching method integrated in the IPM, even though the IPM is orthogonal to the specific matching method.

Moreover, we observe that a moderate number of outer loops have usually been sufficient to significantly improve the matching quality. However and interestingly, the IPM does not guarantee a precision improvement in every outer loop. There are cases in which the matching found by the first outer loop sometimes becomes less accurate than the initial matching. A possible explanation is that the matching problem is non-convex, and the IPM sometimes could not find a better result in one 


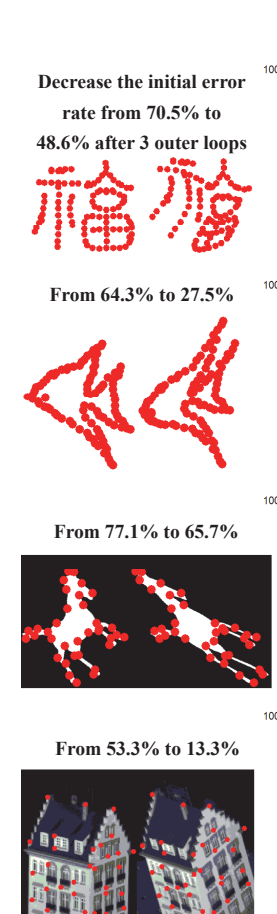

( a )
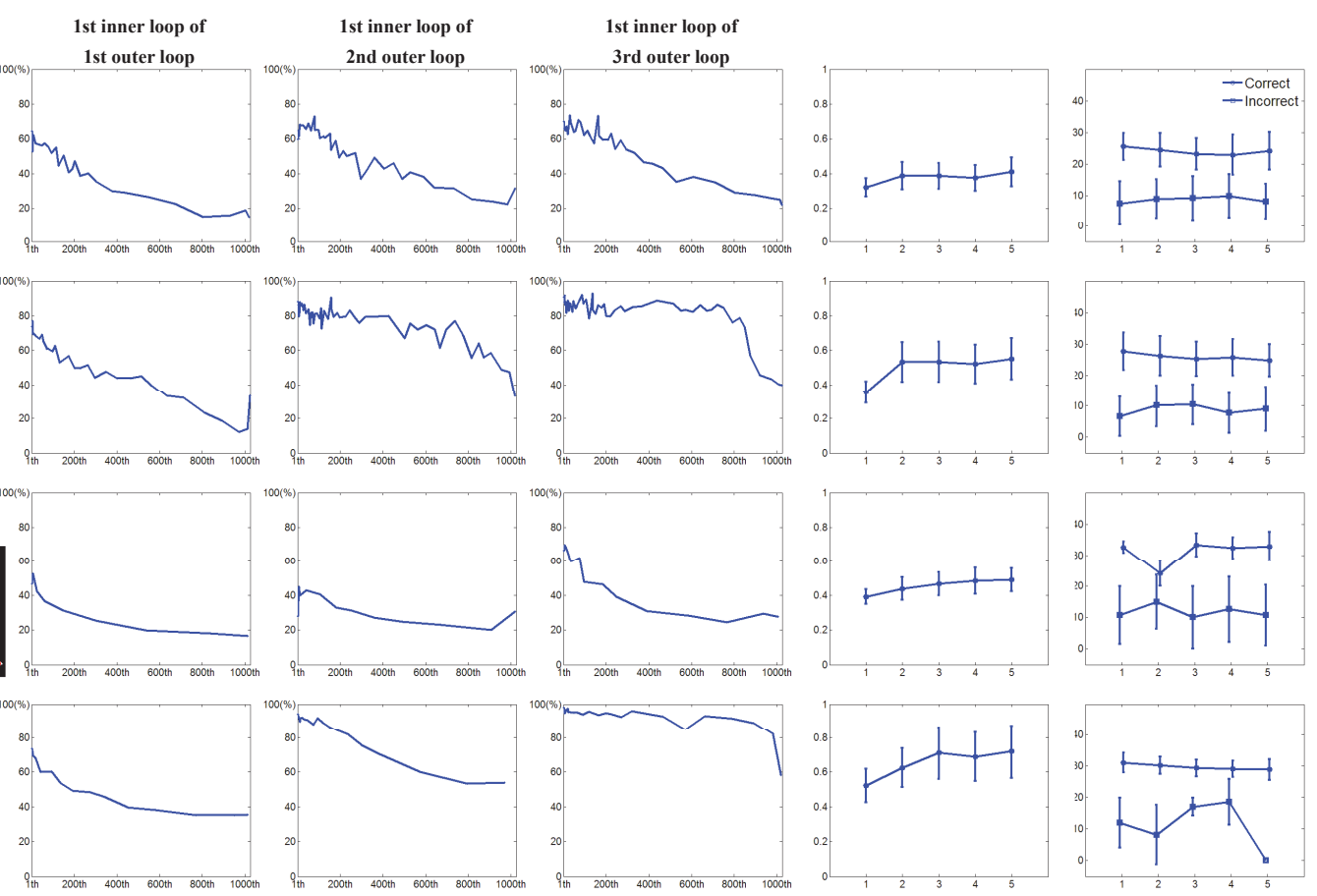

(b)

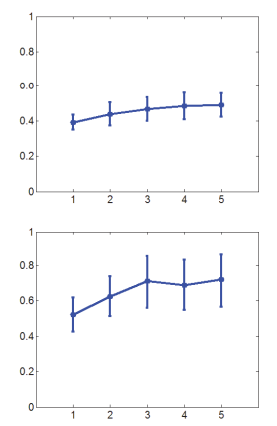

(c)
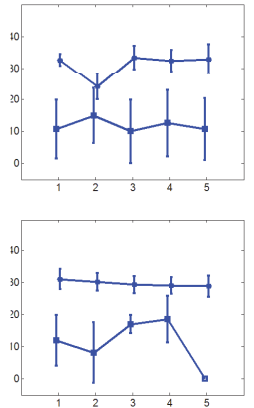

(d)

Figure 3: (a) shows the point sets to be matched; (b) shows the ratio of correct correspondences induced by LCSes, where LCSes are ranked according to the descending order of their lengthes (the $x$-axes indicate the ranking indexes of LCSes). Each outer loop starts with the previous matching and consists of multiple inner loops. In each inner loop, unreliable correspondences are identified and re-matched. Once there is no unreliable correspondence, the outer loop ends and a new matching is obtained; (c) shows the averages and standard deviations of lengthes (normalized to the number of points) of the top-40 LCSes in the first five outer loops; (d) shows the numbers of ballots (with standard deviations) received respectively by correct and incorrect correspondences in the first five outer loops.
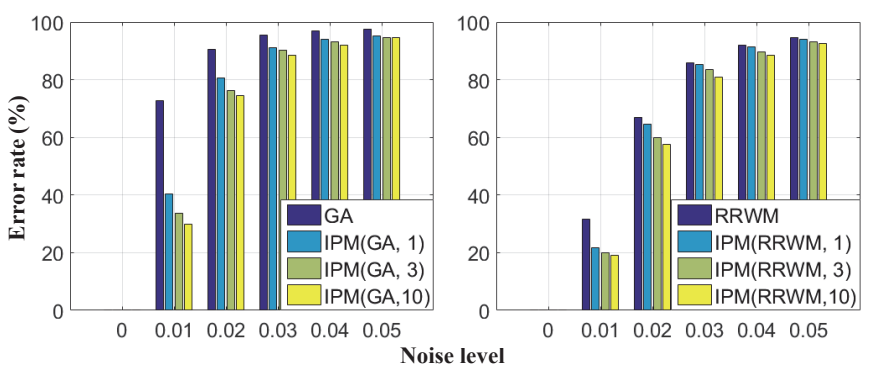

Figure 7: Error rates on the fish-noise sub-dataset.

outer loop. We alleviate this problem by selecting the best everseen intermediate matching (in terms of the matching energy) as the final result. In this case, the IPM would eventually output a better matching than the initial one after a decent number of outer loops. For example, on matching problems having the largest degree of deformation in the Chinese characterdeformation sub-dataset, the average error rate of RRWM is initially $58.5 \%$, then slightly increases to $59.8 \%$ after a single outer loop of IPM (integrating RRWM), and drops to $56.2 \%$ after 3 outer loops, and $52.7 \%$ after 10 outer loops.

\subsubsection{PASCAL Image Dataset}

Here we evaluate the IPM on the PASCAL image dataset. In order to assess the robustness of the IPM against outliers, we pollute two images in each image pair with the same number of outliers. We progressively increase the number of outliers in different matching problems. To be specific, the number of outliers added to each car image increases from 0 to 10 with step size 2, and the number of outliers added to each motorbike image increases from 0 to 20 with step size 2 .

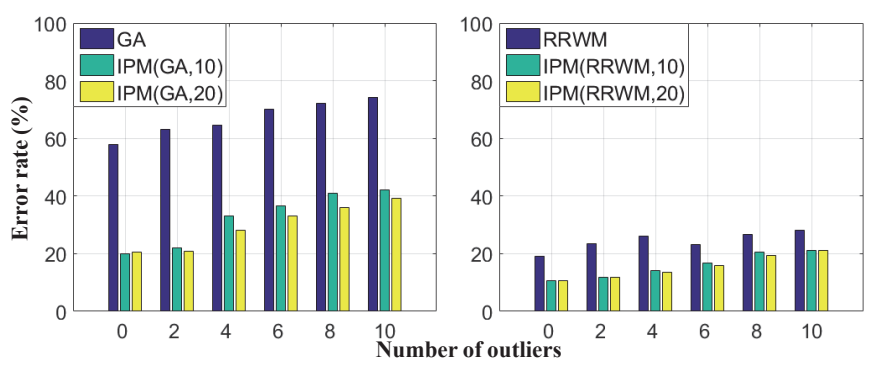

Figure 8: Error rates on the car sub-dataset.

We report the results in Figs. 8 and 9. As we have done in previous experiments, we respectively embed the two base matching methods in the IPM framework $(\alpha=20)$, and observe that the error rate of the IPM often increases when images are polluted with a larger number of outliers. Theoretically, outliers cannot change the order between any pair of feature points. However, they do affect the base matching method integrated in IPM since they change the graph structures to be matched. 


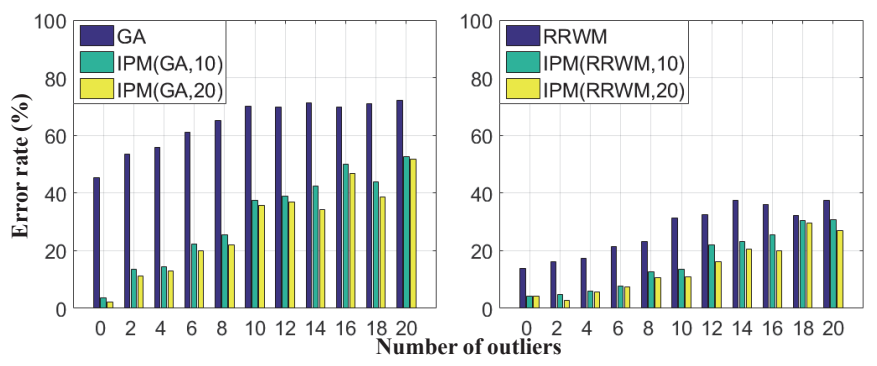

Figure 9: Error rates on the motorbike sub-dataset.

\subsubsection{Horse Silhouette Dataset}

We evaluate the IPM on the horse silhouette dataset [22]. This dataset includes a sequence of 299 consecutive frames, which are generated by gradually shearing a horse silhouette horizontally to twice its width. We extract each frame pair via taking two frames separated by a specific number of in-between frames (which is called the separation between frames hereinafter), and deem it as a graph matching problem. In order to construct matching problems with different degrees of shearing, we progressively increase the separation between frames from 10 to 100 with step size 10 . Here we build the graph model of each frame using Euclidian distances among feature points, thus inevitably induce certain degrees of non-rigid deformation$\mathrm{s}$ to those graph matching problems.

Interestingly, IPM exhibits promising ability of resisting shearing, even when it integrates a base matching method that is vulnerable to shearing. As we can observe from Fig. 10, the IPM significantly reduces the error rate of each base matching method. Even for the most challenging matching problems constructed under the largest separation between frames (i.e., 100 frames), IPM still reduces the average error rates of RRWM by $31.6 \%$.

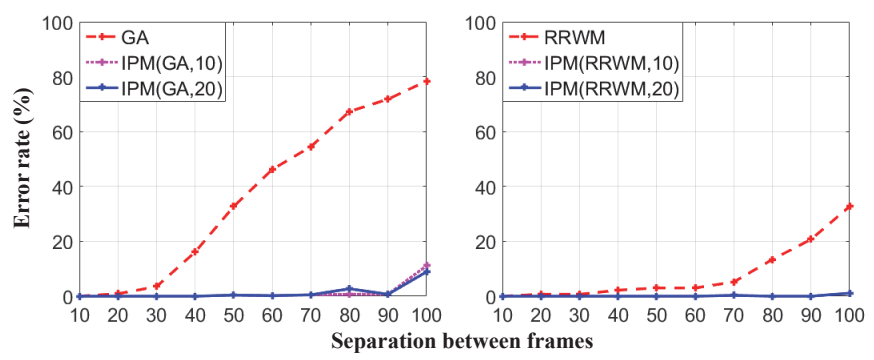

Figure 10: Error rates on the horse silhouette shearing dataset. The separation between frames indicates the degree of shearing.

\subsubsection{D Face 50 Dataset}

We empirically evaluate the IPM on 3D matching problems collected from the face 50 dataset. This dataset consists of 50 point sets, each of which includes 392 points with non-rigid deformations. We randomly select one point set as the template (see Fig. 11-(a)) and match it to all the rest point sets.

The problem size of matching problems in this dataset is larger than that in other datasets studied here. To efficiently com- pute the matching, we set $r=0.1$ in the IPM. In other words, we iteratively reduce the threshold $l$ by one, until there have been more than $0.1 n_{t s}$ correspondences receiving more than $l$ ballots, where $n_{t s}$ is the total number of correspondences.

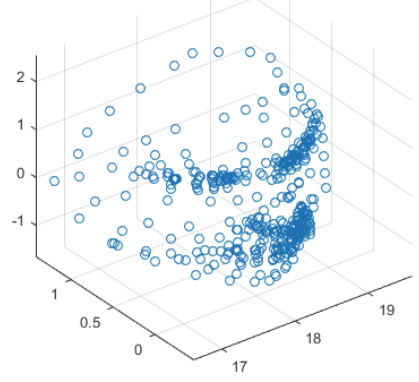

( a )

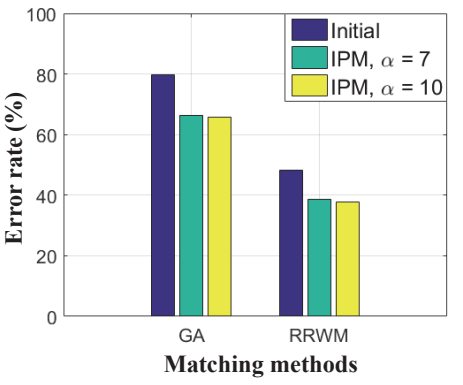

(b)
Figure 11: (a) The template point set. (b) Error rates on the 3D Face 50 dataset.

As shown in Fig. 11-(b), the IPM demonstrates its ability in handling 3D point matching problems with a relatively large problem size. With 10 outer loops, the IPM reduces the average error rate of GA from $79.6 \%$ to $65.8 \%$.

\subsubsection{Synthetic 3D Dataset}

Following the settings in Section 4.2.4 ( $r=0.1)$, we evaluate the effectiveness of the IPM in dealing with $3 \mathrm{D}$ matching problems with noise pollution. We generate 2000 pairs of point sets. Every 500 pairs have the same level of noise pollution. Specifically, in each pair of point sets, one point set includes 100 points that are uniformly distributed in a 1 by 1 by 1 cube. The other point set is generated by polluting the first point set with a normally distributed noise conforming to $N\left(0, \sigma^{2}\right)$. We increase $\sigma$ from 0.01 to 0.04 with step size 0.01 , and match 500 pairs of point sets for each $\sigma$. We present the average error rates in Fig. 12. We observe that the IPM significantly decreases the error rates of the tested base matching methods.

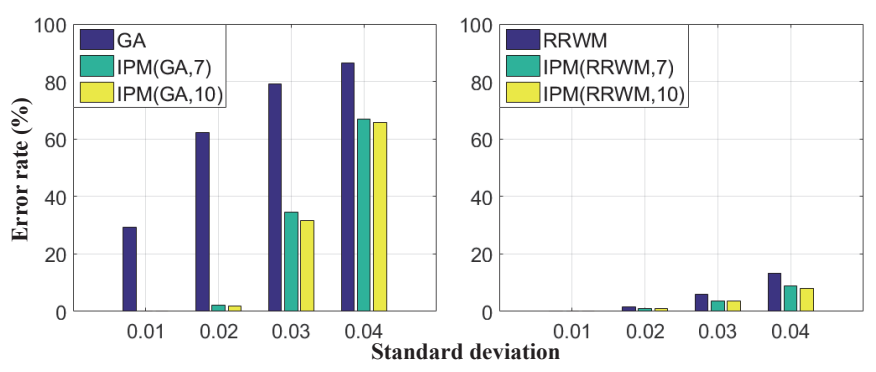

Figure 12: Error rates on the synthetic 3D dataset.

\subsubsection{CMU Hotel Sub-dataset}

We create frame pairs as we have done in Section 4.2.3, using this sub-dataset. The deformation between two frames in each pair can be approximately deemed to be rigid. We vary the separation between frames from 10 to 90 with step size 10 , and 


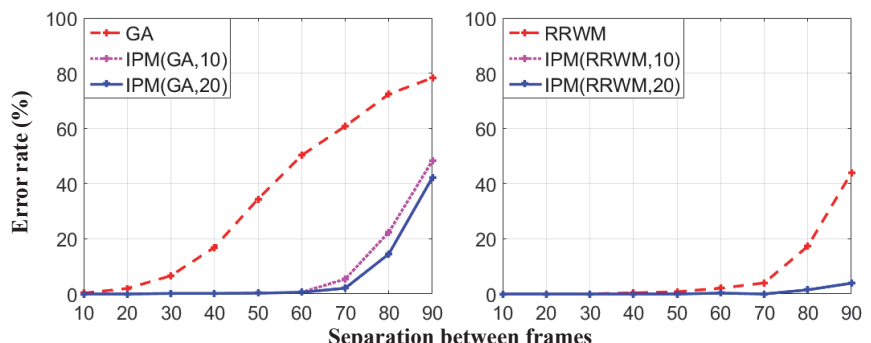

Figure 13: Error rates on the CMU hotel sub-dataset.

evaluate the IPM and base matching methods on the obtained matching problems.

We report experimental results in Fig. 13, and observe that the IPM significantly reduces the matching error rate of each base matching method, even when the initial matching has a large error rate (e.g., error rate higher than 50\%). This benefit of IPM can mainly be attributed to the novel MGS and RC$\mathrm{S}$ mechanisms which collaboratively identify unreliable point correspondences. Iteratively identifying and re-matching unreliable correspondences eventually lead to the better matching precision of IPM.

\subsubsection{Time Complexity}

After evaluating the precision improvements, we analysis the time complexity of IPM here. Given two point sets having $n_{t}$ and $n_{s}$ feature points (respectively), the time complexity of MGS is $O\left(n_{t} \lg n_{t}+n_{s} \lg n_{s}\right)$, and the time complexity of RCS is $O\left(n_{t} n_{s}\right)$. The time complexity of the whole IPM depends on the parameter $r$ specifying the lowest percentage of correspondences that must be identified as reliable ones in each inner loop (i.e., at least $r n_{t s}$ correspondences must be identified as reliable ones in each inner loop). This parameter controls the trade-off between matching quality and computational efficiency. To be specific, when $r$ is larger than 0 , every outer loop contains at most $\left[\frac{1}{r}\right]$ inner loops. As a result, the time complexity of IPM is of the same order with the integrated base matching method (see Algorithm 3). With a larger $r$, more correspondences will be identified as reliable ones in each inner loop, and the IPM has better computational efficiency as the total number of inner loops decreases. When $r=0$, the IPM focuses on the matching quality rather than the efficiency. The worst-case time complexity of IPM becomes higher than (thus dominates) that of the base matching method integrated in the IPM by an order of magnitude. Yet the computation time of the IPM with $r=0$ is still acceptable in most cases. To be specific, we record the computation time of the IPM ( $r=0, \alpha=10)$ over the CMU house sub-dataset including 279 pairs of frames and report the results in Table 1.

This experiment is conducted in a regular desktop with $2 \mathrm{G}$ RAM and 3.2GHz Intel(R) Core(TM) i5-3470 CPU. The IPM is implemented with Matlab and $C$. The IPM takes roughly $0.5 \mathrm{~s}$ on average to match a frame pair, which demonstrates its efficiency. The IPM can be further accelerated by parallelizing the MGS and RCS mechanisms.

Table 1: Computation Time (unit: second)
\begin{tabular}{|c|c|c|}
\hline & GA & RRWM \\
\hline Initial matching \& Re-matching & 0.21 & 0.39 \\
\hline MGS \& RCS & 0.19 & 0.18 \\
\hline IPM & 0.45 & 0.62 \\
\hline
\end{tabular}

\section{Conclusion}

In this paper, we mainly focus on improving the point matching precision via iteratively applying classical graph matching methods. The initial matching is improved by the proposed IPM framework with two nested loops. In each inner loop, two novel mechanisms, MGS and RCS, which are invariant to scaling and translation, are applied to find reliable correspondences. These reliable correspondences contribute to the re-matching process of the rest feature points. In each outer loop, a new intermediate matching is obtained. The best intermediate matching is selected as the final result. Extensive experiments demonstrate that the improvements achieved by IPM are significant.

In our future work, we would like to study the extended framework of IPM that dynamically and adaptively selects suitable graph matching methods in re-matching processes. Moreover, we would like to further expand the application scope of IPM to make it suitable for other point matching methods.

\section{Appendix A. Proof of Porperty 1}

We apply the method of proof by contradiction here. If $X^{*}$ is not the global optimum of our energy function, there must be another $X^{\prime}$ with lower energy

$$
\mathbf{x}^{\prime T} H^{k k^{\prime}} \mathbf{x}^{\prime}-w \sum_{i=1}^{n_{t}} \sum_{j=1}^{n_{s}} X_{i j}^{\prime}<\mathbf{x}^{* T} H^{k k^{\prime}} \mathbf{x}^{*}-w \sum_{i=1}^{n_{t}} \sum_{j=1}^{n_{s}} X_{i j}^{*},
$$

where $\mathbf{x}^{\prime}$ and $\mathbf{x}^{*}$ are binary variable vectors concatenating column vectors of matrix $X^{\prime}$ and $X^{*}$ respectively. If $\mathbf{x}^{\prime T} H^{k k^{\prime}} \mathbf{x}^{\prime}>$ $\mathbf{x}^{* T} H^{k k^{\prime}} \mathbf{x}^{*}$, based on the constraints on the solution and the definition of $H^{k k^{\prime}}$, we have

$$
\mathbf{x}^{\prime T} H^{k k^{\prime}} \mathbf{x}^{\prime}-\mathbf{x}^{* T} H^{k k^{\prime}} \mathbf{x}^{*} \geq 1 .
$$

By $0<w<\frac{1}{n_{t} n_{s}}$, we know that

$$
w \sum_{i=1}^{n_{t}} \sum_{j=1}^{n_{s}} X^{\prime}{ }_{i j}-w \sum_{i=1}^{n_{t}} \sum_{j=1}^{n_{s}} X^{*}{ }_{i j}<1 .
$$

Hence we have

$$
\mathbf{x}^{\prime T} H^{k k^{\prime}} \mathbf{x}^{\prime}-w \sum_{i=1}^{n_{t}} \sum_{j=1}^{n_{s}} X^{\prime}{ }_{i j}>\mathbf{x}^{* T} H^{k k^{\prime}} \mathbf{x}^{*}-w \sum_{i=1}^{n_{t}} \sum_{j=1}^{n_{s}} X^{*}{ }_{i j},
$$

which contradicts to (A.1). As a result,

$$
\mathbf{x}^{\prime T} H^{k k^{\prime}} \mathbf{x}^{\prime}<\mathbf{x}^{* T} H^{k k^{\prime}} \mathbf{x}^{*},
$$


or

$$
\left\{\begin{array}{l}
\mathbf{x}^{\prime T} H^{k k^{\prime}} \mathbf{x}^{\prime}=\mathbf{x}^{* T} H^{k k^{\prime}} \mathbf{x}^{*} \\
w \sum_{i=1}^{n_{t}} \sum_{j=1}^{n_{s}} X^{\prime}{ }_{i j}<w \sum_{i=1}^{n_{t}} \sum_{j=1}^{n_{s}} X^{*}{ }_{i j} .
\end{array}\right.
$$

We assume that (A.5) is true. According to the definition of LCS, we know that

$$
\mathbf{x}^{* T} H^{k k^{\prime}} \mathbf{x}^{*}=0
$$

Besides, all the elements in $H^{k k^{\prime}}$ are non-negative, so

$$
\mathbf{x}^{\prime T} H^{k k^{\prime}} \mathbf{x}^{\prime} \geq 0 \text {. }
$$

(A.5), (A.7) and (A.8) lead to a contradiction. Thus we assume that (A.6) is true. Combining (A.6) and (A.7), We obtain that

$$
\mathbf{x}^{T} H^{k k^{\prime}} \mathbf{x}^{\prime}=0 .
$$

(A.6) and (A.9) suggest that $X^{\prime}$ includes more correspondences between real feature points than those in $X^{*}$ while the correspondences in $X^{\prime}$ do not violate each other. Thus, $X^{\prime}$ leads to another common subsequence which is longer than the LCS. This is a contradiction to Definition 5. Hence Property 1 is proved.

\section{References}

[1] R.G. Bartle, D.R. Sherbert, Introduction to real analysis, volume 2, Wiley, 1992.

[2] S. Belongie, J. Malik, J. Puzicha, Shape matching and object recognition using shape contexts, IEEE Trans. Pattern Anal. Mach. Intell. 24 (2002) 509C522.

[3] P.J. Besl, N.D. McKay, Method for registration of 3-d shapes, in: Robotics-DL tentative, International Society for Optics and Photonics, pp. 586C606.

[4] T.M. Breuel, A comparison of search strategies for geometric branch and bound algorithms, in: Eur. Conf. Comput. Vis., Springer, 2002, pp. $837 \mathrm{C} 850$.

[5] T.S. Caetano, J.J. McAuley, L. Cheng, Q.V. Le, A.J. Smola, Learning graph matching, IEEE Trans. Pattern Anal. Mach. Intell. 31 (2009) $1048 \mathrm{C} 1058$.

[6] M. Cho, J. Lee, K.M. Lee, Reweighted random walks for graph matching, in: Eur. Conf. Comput. Vis., Springer, 2010, pp. 492C505.

[7] M. Cho, K.M. Lee, Progressive graph matching: Making a move of graphs via probabilistic voting, in: Proc. IEEE Conf. Comput. Vis. Pattern Recognit., IEEE, pp. 398C405.

[8] H. Chui, A. Rangarajan, A new point matching algorithm for nonrigid registration, Computer Vision and Image Understanding 89 (2003) $114 \mathrm{C} 141$.

[9] D. Conte, P. Foggia, C. Sansone, M. Vento, Thirty years of graph matching in pattern recognition, Int. J. Pattern Recogn. 18 (2004) 265 C298.

[10] T.H. Cormen, C.E. Leiserson, R.L. Rivest, C. Stein, et al., Introduction to algorithms, volume 2, MIT press Cambridge, 2001.

[11] T. Cour, P. Srinivasan, J. Shi, Balanced graph matching, NIPS, volume 2, p. 6.

[12] M. Everingham, L. Van Gool, C.K. Williams, J. Winn, A. Zisserman, The pascal visual object classes (voc) challenge, Int. J. Comput. Vision 88 (2010) 303C 338.

[13] M.A. Fischler, R.C. Bolles, Random sample consensus: a paradigm for model fitting with applications to image analysis and automated cartography, Commun. ACM 24 (1981) 381C395.

[14] A.W. Fitzgibbon, Robust registration of $2 \mathrm{~d}$ and $3 \mathrm{~d}$ point sets, Image and Vision Computing 21 (2003) 1145C1153.

[15] S. Gold, A. Rangarajan, A graduated assignment algorithm for graph matching, IEEE Trans. Pattern Anal. Mach. Intell. 18 (1996) 377C388.

[16] P.J. Huber, Robust statistics, Springer, 2011.
[17] B. Jian, B.C. Vemuri, Robust point set registration using gaussian mixture models, IEEE Trans. Pattern Anal. Mach. Intell. 33 (2011) 1633C1645.

[18] H. Jiang, M.S. Drew, Z.N. Li, Matching by linear programming and successive convexification, IEEE Trans.PatternAnal.Mach.Intell. 29 (2007) 959C975.

[19] D.T. Lee, B.J. Schachter, Two algorithms for constructing a delaunay triangulation, International Journal of Computer $\mathcal{E}$ Information Sciences 9 (1980) 219C242.

[20] M. Leordeanu, M. Hebert, A spectral technique for correspondence problems using pairwise constraints, in: Proc. IEEE Int. Conf. Comput. Vis., volume 2, pp. 1482 C1489.

[21] M.Leordeanu, M.Hebert, R.Sukthankar, An integer projected fixed point method for graph matching and map inference, in: Proc. Conf. Neural Information Processing Systems, pp. 1114C1122.

[22] H. Li, X. Huang, L. He, Object matching using a locally affine invariant and linear programming techniques, IEEE Trans. Pattern Anal. Mach. Intell. (2013) 411C424.

[23] X. Li, Z. Hu, Rejecting mismatches by correspondence function, Int. J. Comput. Vision 89 (2010) 1 C17.

[24] A. Myronenko, X. Song, Point set registration: Coherent point drift, IEEE Trans. Pattern Anal. Mach. Intell. 32 (2010) 2262C2275.

[25] S. Park, M. Hebert, Fast and scalable approximate spectral matching for higher-order graph matching, IEEE Trans. Pattern Anal. Mach. Intell. (2013).

[26] P.J. Rousseeuw, Least median of squares regression, Journal of the American statistical association 79 (1984) 871C880.

[27] C. Scott, R. Nowak, Robust contour matching via the order-preserving assignment problem, IEEE Trans. Image Process. 15 (2006) 1831C1838.

[28] L. Torresani, V. Kolmogorov, C. Rother, A dual decomposition approach to feature correspondence, IEEE Trans. Pattern Anal. Mach. Intell. 35 (2013) 259C271.

[29] Y. Tsin, T. Kanade, A correlation-based approach to robust point set registration, in: Eur. Conf. Comput. Vis., Springer, 2004, pp. 558C569.

[30] R. Zass, A. Shashua, Probabilistic graph and hypergraph matching, in: Proc. IEEE Conf. Comput. Vis. Pattern Recognit., pp. 1 C8.

[31] J. Zhao, J. Ma, J. Tian, J. Ma, D. Zhang, A robust method for vector field learning with application to mismatch removing, in: Proc. IEEE Conf. Comput. Vis. Pattern Recognit., IEEE, pp. 2977 C2984.

[32] Y. Zheng, D. Doermann, Robust point matching for nonrigid shapes by preserving local neighborhood structures, IEEE Trans. Pattern Anal. Mach. Intell. 28 (2006) 643C649.

[33] F. Zhou, F. De la Torre, Factorized graph matching, in: Proc. IEEE Conf. Comput. Vis. Pattern Recognit., pp. 127C134. 


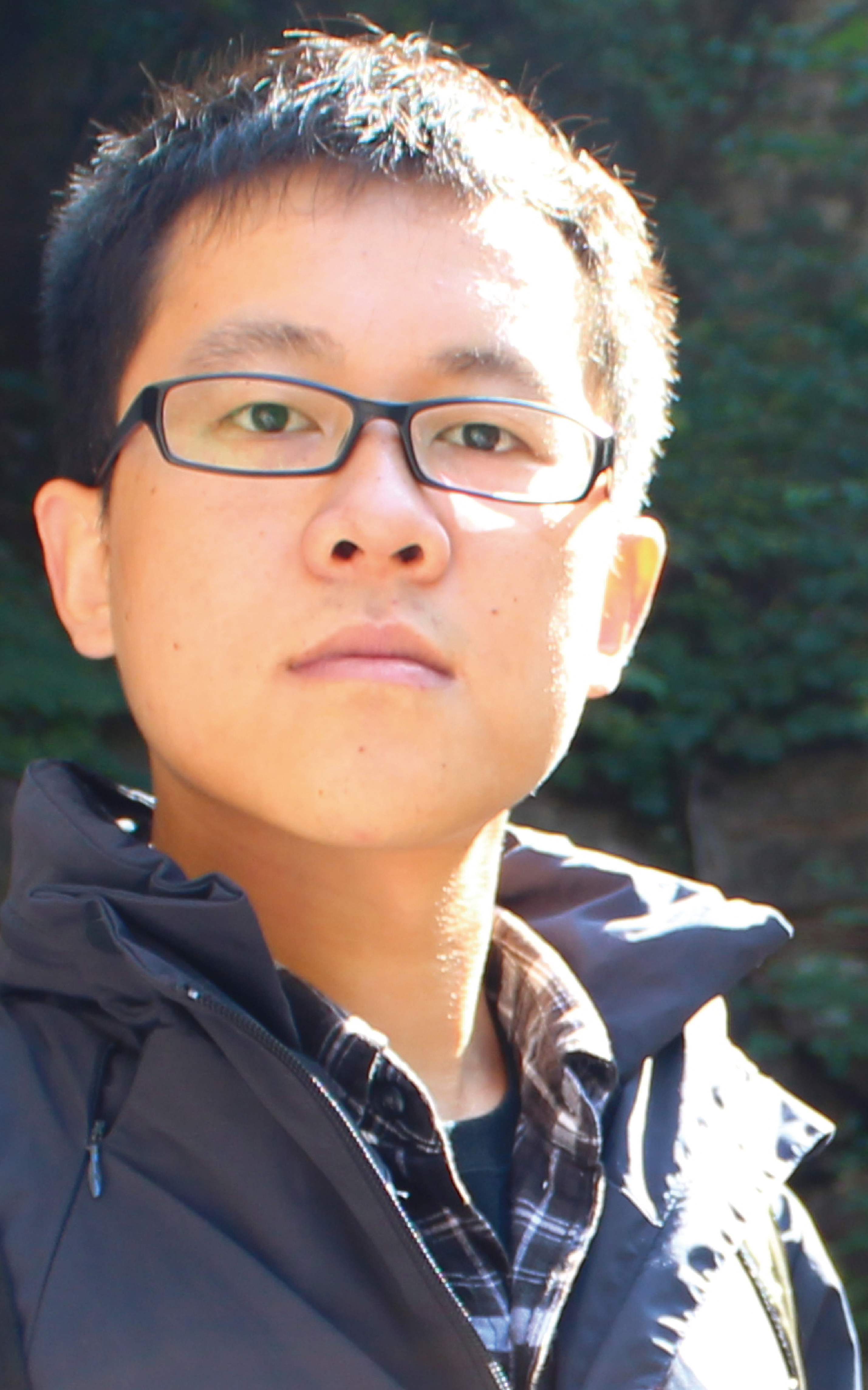




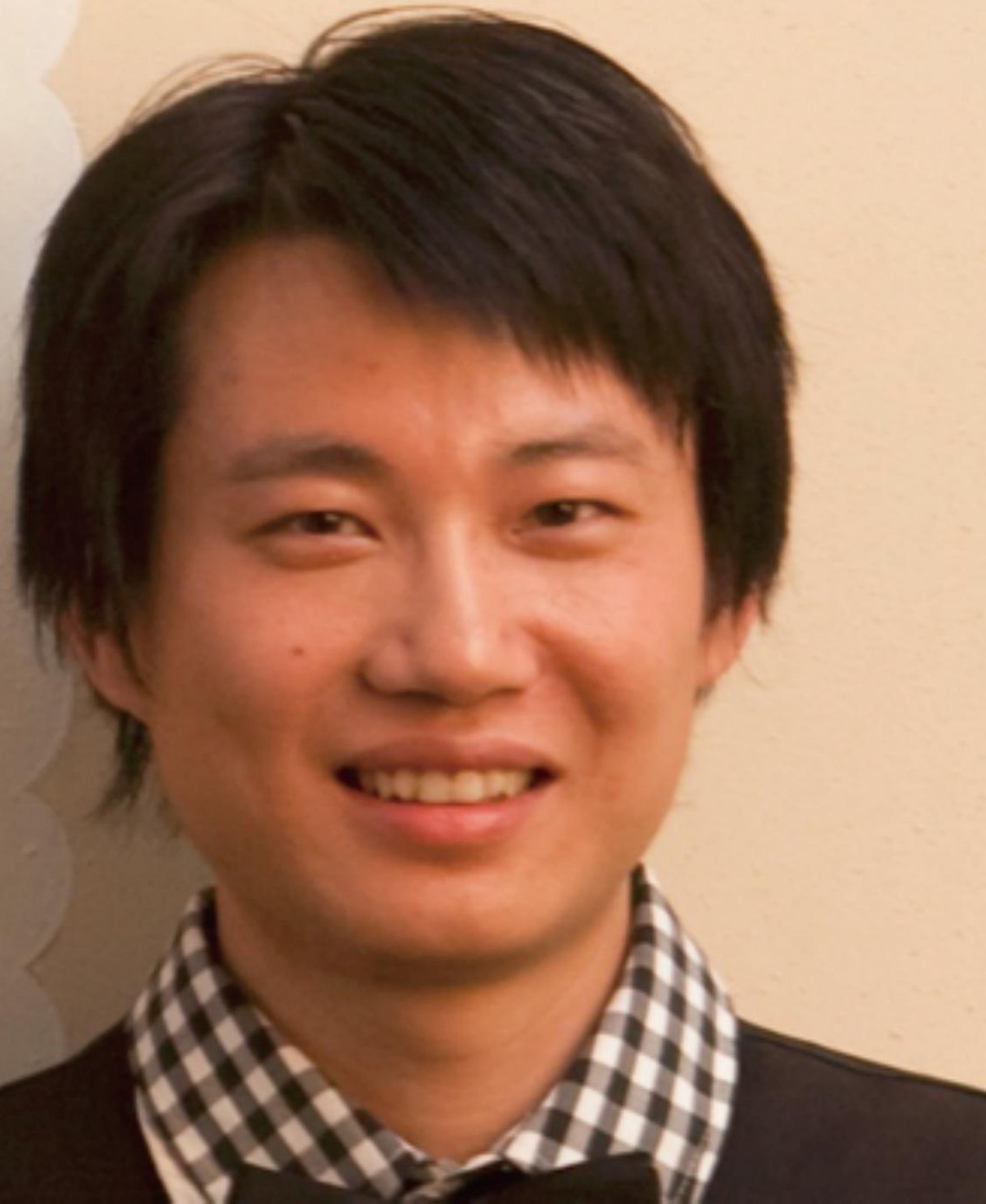



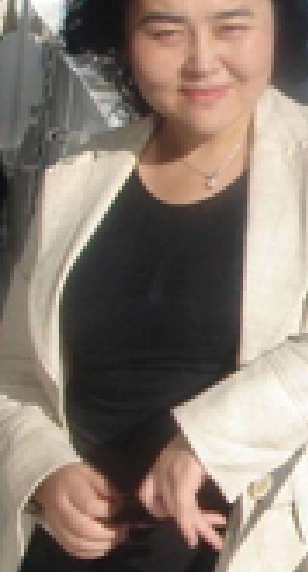


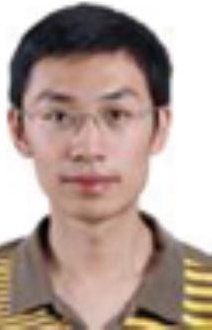

